\section{Support-Based and ML Approaches to DOA Estimation in a Dumb Sensor Network}

\author{
Stefano Marano, Vincenzo Matta, Peter Willett, Fellow, IEEE, and \\ Lang Tong, Fellow, IEEE
}

\begin{abstract}
A recent paper by Marano $e$ al . shows that a network of unconnected and completely direction-of-arrival (DOA)-blind sensors ("beepers") is able to perform DOA estimation quite effectively within the SENMA architecture (unlabeled polling performed by a mobile agent). The idea is that the mobile agent collects the periodic emissions of the polled sensors, with the time origin of such emissions being the passage of the acoustic wavefront. Depending on the relative orientation between the acoustic wavefront and the field of view of the mobile agent, the impinging times over different sensors are more or less clustered and so are the recorded emissions. On this basis, the DOA may be inferred. Here, two new estimators are proposed. One method (support-based) exploits the maximum spread between recorded times and is simple to implement, and its performance, measured in terms of mean square error, is improved significantly versus that proposed in the recent paper by Marano et al. In fact, the support-based estimator achieves performance close to that of the maximum-likelihood (ML) estimator-also investigated here-indicating that the support-based structure is perhaps suitable for tasks that involve cheap robust designs, such as sea/ground surveillance and sniper location.
\end{abstract}

Index Terms-Direction-of-arrival (DOA) estimation, maximum likelihood, wireless sensor networks.

\section{INTRODUCTION AND BASIC MODEL}

In a recent paper [1], we addressed the problem of estimating the direction of arrival (DOA) of a remotely generated plane wave via a wireless network of small, inexpensive, randomly located and possibly unreliable sensors. The positions of the sensors are unknown and they do not communicate with each other: A Poisson field of working sensors was the basis. We assume that mobile agents travel across the area and poll the sensors to collect information useful to the DOA estimation. Such a network architecture, known as SENMA (sensor networks with mobile agents) [2], has recently attracted increasing interest. One of its features is the parsimony with energy versus alternative architectures such as ad hoc multihop flat wireless networks.

Key assumptions of our model are that the sensors' antennas are isotropic (individually they are completely blind to the DOA) and that, on the other hand, the mobile agents (rovers) must use directional fields of view in their interrogations of these sensors. In their "walks" across the surveyed area, the agents take successive uncorrelated dwells (henceforth snapshots) of sensors inside their fields of view that (for simplicity of analysis) are taken as elliptical. For the sake of concreteness, let us assume that the goal is to estimate an acoustic DOA (so that the sensors have acoustic receiving antennas), while the communications between sensors and mobile agents is electromagnetic. We shall discuss these assumptions further when we summarize.

Manuscript received December 9, 2004; revised May 16, 2005. This work was partially supported by the Office of Naval Research. The associate editor coordinating the review of this manuscript and approving it for publication was Dr. Feng Zhao.

S. Marano and V. Matta are with the Department of Electrical and Information Engineering, Università degli Studi di Salerno, I-84084 Fisciano (SA), Italy (e-mail: marano@unisa.it; vmatta@unisa.it).

P. Willett is with the Electrical and Computer Engineering Department, University of Connecticut, Storrs, CT 06269 USA (e-mail: willett@ engr.uconn.edu)

L. Tong is with the Electrical and Computer Engineering, Cornell University, Ithaca, NY 14853 USA (e-mail: 1tong@ece.cornell.edu).

Digital Object Identifier 10.1109/TSP.2006.870553

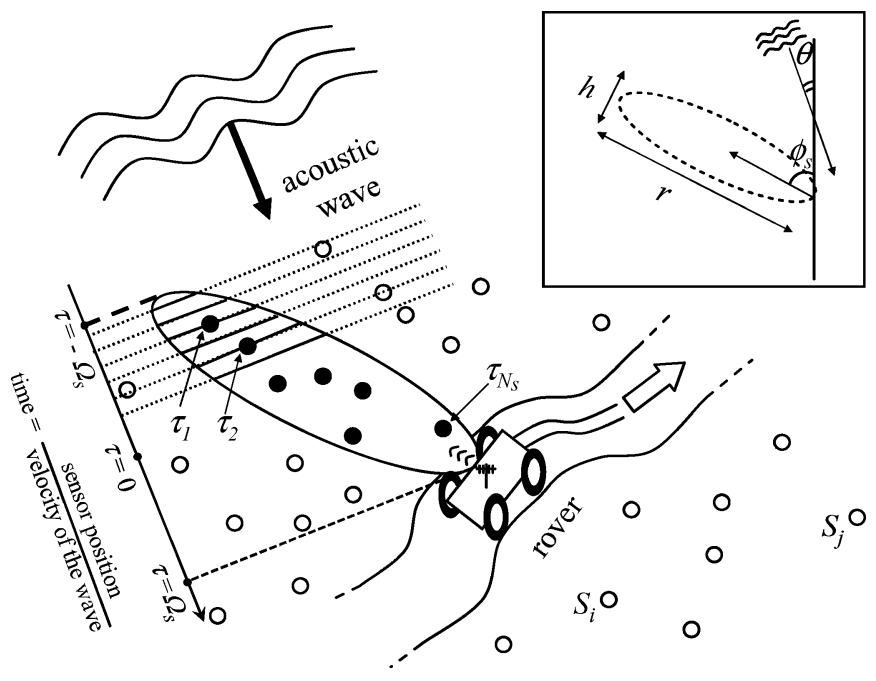

Fig. 1. Scenario: A traveling rover polls the remote sensors $\mathcal{S}_{k}$ lying inside its field of view. The dotted lines represent the wavefront at successive times: $\tau_{1}$ and $\tau_{N_{S}}$ are the smaller and the larger impinging times over the $N_{s}$ polled sensors. The bold lines are the chords that the wavefront intercepts over the ellipse; their lengths are relevant to the impinging-time density estimation of (1) and (2). Also shown is the system reference for the impinging times. We see that $\tau_{i} \in\left(-\Omega_{s}, \Omega_{s}\right)$ and that $\Omega_{s}$ depends on the relative orientation between DOA and rovers' field of view. In the separate box, some notation is made explicit: $\theta$ is the desired DOA and $\phi_{s}$ is the rover's orientation at snapshot $s$; the rover's field of view is an ellipse with major and minor axes $r$ and $h$.

The reference scenario is depicted in Fig. 1, which reproduces, to some extent, Fig. 1 of [1]. The unknown $\mathrm{DOA}^{1}$ is $\theta \in(0, \pi)$, and the rover orientation at step $s$ is defined by the angle $\phi_{s} \in(0,2 \pi)$; this is assumed known to the signal processing center, which may be thought of as sited aboard the rover. The sensors' locations are unknown, and their number is modeled according to a Poisson field law: The probability of finding $m$ sensors inside an arbitrarily shaped region of area $A$ is $(\lambda A)^{m} \exp \{-\lambda A\} / m$ !, with $\lambda$ being the average number of sensors per unit area. The Poisson assumption is reasonable on physical grounds: It follows from assuming that 1) no two sensors may be located at the same point, 2) the probability of finding one sensor in an elementary area $\Delta_{A}$ is roughly proportional (through $\lambda$ ) to $\Delta_{A} ; 3$ ) the probability of finding in $\Delta_{A}$ more than one sensor vanishes faster than $\Delta_{A}$; and 4) the process of sensor counting has independent and stationary increments (for a formal statement, see, e.g., [3]). Such conditions uniquely imply the Poisson distribution that is critical to the technique of [1]; however, although a Poisson law does form the basis for performance comparison, the estimators here do not require it: All that is needed in this paper is that the reporting sensors be uniformly distributed within the rover's field of view.

Let $\tau_{i}$ denote the time instant that the $i^{t h}$ sensor, say $\mathcal{S}_{i}$, meets a planar acoustic wave. From then on, it emits an (electromagnetic) periodic waveform $x_{i}(t)=\sum_{k} p\left(t-\tau_{i}-k T\right)$, for some short basic pulse $p(t)$ and some prescribed time period $T$ : Essentially, the sensor beeps, although for reasons of battery life and confusion, these beeps ought to be only virtual, until polled by the rover. The period $T$ must not be smaller than $2 r / v$, with $v$ being the speed of the acoustic wavefront in the medium and $r$ the maximum distance at which the rover may sense the remote devices (for us, this is the major axis of the ellipse).

${ }^{1}$ The reason why we do not define $\theta \in(0,2 \pi)$ is that our methods are unable to resolve an ambiguity of $\pi$ : the two DOAs $\theta$ and $\theta+\pi$ are absolutely undistinguishable. 

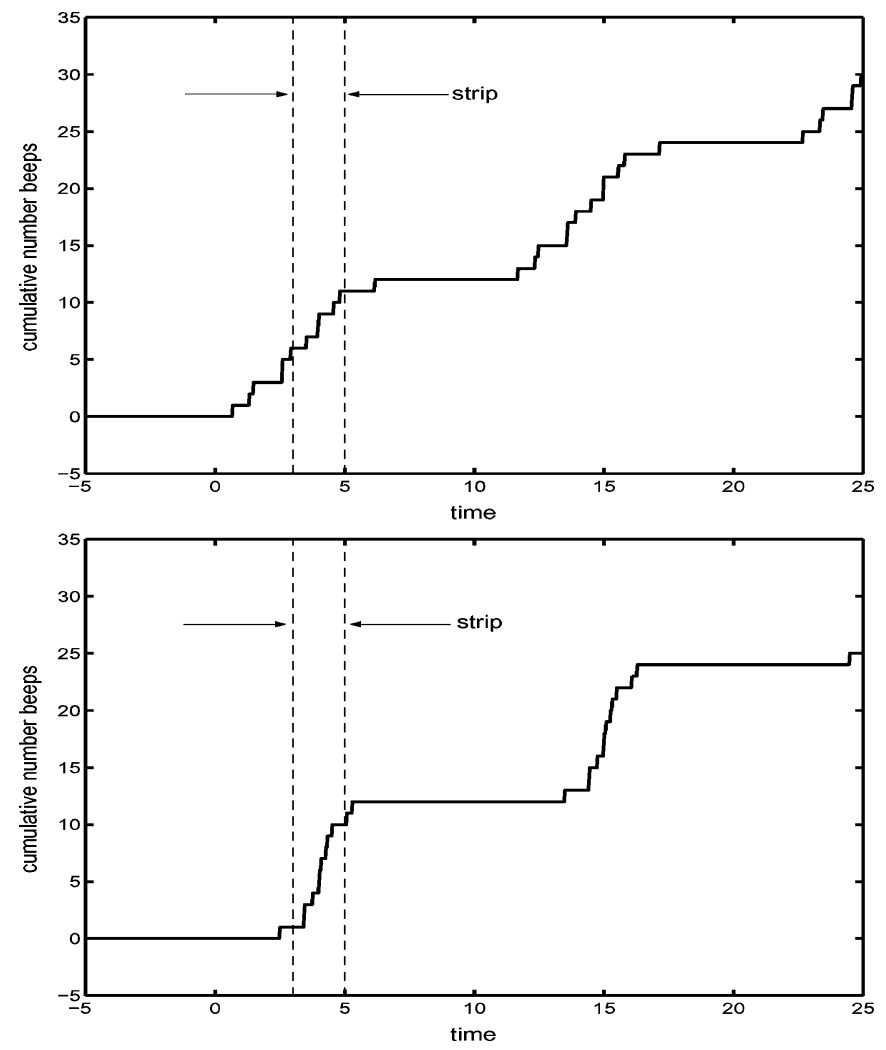

Fig. 2. Scheme of [1]: The cumulative number of "beeps" heard by the rover as a function of time, for a source that is (top) endfire from the rover's elliptical field of view (i.e., the plane wave travel's along the ellipse's major axis) and (bottom) broadside. The orientation, according to [1] (improved on in this correspondence), can be estimated from the number of beeps in the indicated "strip" of time.

The (ellipsoidal) rover's field of view is defined as the region such that all the sensors lying inside it are seen by the rover, while sensors lying outside are invisible. Accordingly, the signal collected by the rover is the aggregate of a certain number $N_{s}$ of waveforms: $r_{s}(t)=$ $\sum_{i=1}^{N_{s}} x_{i}(t)$. The signal is recorded for an appropriate time interval to allow the rover to order the received pulses so that $\tau_{i} \leq \tau_{i+1}$, $i=1,2, \ldots, N_{s}-1$ [1]. Now consider Fig. 2, which compares a beep-train from a plane-wave-oriented "endfire" to the rover's elliptical field of view (i.e., the plane wave travels along the ellipse's major axis) to a "broadside" impingement: The ensemble of sensors lying in the field of view encounter the wavefront for times that are, respectively, comparatively long and short. Thus, there is a greater beep concentration in the latter than the former situation. A first investigation of this problem [1] suggested measuring this concentration by counting the number of returns (beeps) near the central region (the strip in Fig. 2). In fact, despite this idea's simplicity, DOA estimation is both possible and reliable: asymptotic efficiency, as measured in terms of the Fisher information, ${ }^{2}$ is attained in a moderate number of snapshots. However, certainly information is sacrificed, and in this correspondence, we use fuller information to capture not only the concentration of beeps, but also the shape of their time distribution.

Now, note that there is no possibility of association between the times $\tau_{i}$ and sensor position inside the rover lobe (meaning its field of view); although for purposes of estimation, it would be convenient to know from where each beep came, that would be incompatible with our assumptions of a random (Poisson) field, broadcast polling (within the

${ }^{2}$ The Fisher information is computed using only the number of beeps, not the actual vector of beep times, nor time differences. rover's field of view), broadcast beeping, and of the essential dumbness of the sensors. Further, we shall avoid any use of the absolute time of sensors' beeps, and use only the relative interbeep times to help in our estimation; as the identity and location of each sensor is not known to the rover, the information loss incurred is minimal. That is, we observe at snapshot $s$ the ordered vector of times $\tau_{s}=\left(\tau_{1}, \tau_{2}, \ldots, \tau_{N_{s}}\right)$, but in fact, our data is only the $N_{s}-1$ relative times taken with respect to (say) $\tau_{1}: t_{s}=\left(t_{2}, t_{3}, \ldots, t_{N_{s}}\right)$, with $t_{i}=\tau_{i}-\tau_{1}, \forall i$.

The aim of this correspondence is to investigate more optimal inference procedures. As in the previous paper [1], it is assumed that successive snapshots taken by the rover result in independent observations - a condition easily obtainable (at least approximately) in practice - and that there is variety in the orientation of the rover's field of view among these snapshots.

In Section II, we propose an easy implementable estimator based on a "telescoping" of support intervals, while Section III deals with the (optimal) maximum-likelihood (ML) approach. The main results are illustrated in Section IV where, as expected, it is seen that former estimator suffers from comparison to the latter in terms of performance; however, it is much simpler and faster. Further, there is apparently a significant gain versus the scheme of [1]. Final discussions and a summary of the main findings are offered in Section V. Some mathematics are postponed in an Appendix.

\section{SUPPORT-BASED DOA ESTIMATION}

To gain insight, consider again Fig. 1, but imagine that a continuum of sensors lies within the surveyed area. Then, for given DOA $\theta$ and rover orientation $\phi_{s}$ (recall that the subscript $s$ is the snapshot number), it makes sense to define the density of the vector $\tau_{s}$ and accordingly consider the measured times (the $\tau_{i}{ }^{\prime}$ 's) as independent samples from the implied probability density function (pdf). This latter can be easily derived: From Fig. 1, we see that the density of sensors corresponding to (for instance) delay $\tau_{2}$ is proportional to the length of the chord (bold line) formed by the intersection of the acoustic wavefront and the elliptic field of view. Computing these lengths is a simple matter and the final result is

$$
f_{\tau_{i}}\left(\tau ; \Omega_{s}\right)=\left\{\begin{array}{cc}
\frac{2}{\pi \Omega_{s}} \sqrt{1-\left(\frac{\tau}{\Omega_{s}}\right)^{2}}, & -\Omega_{s} \leq \tau \leq \Omega_{s} \\
0, & \text { elsewhere }
\end{array}\right.
$$

wherein the support ${ }^{3}$ is the maximum allowable spread of the measured times. This, as made explicit in Fig. 1, amounts to

$$
\Omega_{s}=\Omega_{s}(\theta)=\frac{1}{2 v} \sqrt{r^{2} \cos ^{2}\left(\theta-\phi_{s}\right)+h^{2} \sin ^{2}\left(\theta-\phi_{s}\right)}
$$

with $v$ the velocity of sound, $r$ and $h$ the two main axes of the ellipse, and $\phi_{s}$ the ellipse orientation at snapshot $s$. Note that for (1) to hold, we must assume an elliptical field of view. If the field of view is not elliptical, then another pdf results, with little effect on our mode of analysis; we choose the ellipse for concreteness only. From (1) and (2), we see that the support of the $\tau_{i}$ density is $\left(-\Omega_{s}, \Omega_{s}\right)$ and depends upon the unknown DOA $\theta$. Specifically, as seen from (2) and illustrated in Fig. 3, the function $\Omega_{s}(\theta)$ is periodic in $\theta$ with period $\pi$ and, for each snapshot $s, \Omega_{s}(\theta)$ shifts (circularly, or modulo- $\pi$ ) of an amount that depends on the current value of $\phi_{s}$. Accordingly, a possibility is to estimate the support- $\Omega_{s}$-and from this to infer $\theta$. Let us see if, and how, this idea works.

We want to estimate the support $\Omega_{s}$. Perhaps the easiest estimator is half the range of the measured times: $\left(\tau_{N_{s}}-\tau_{1}\right) / 2$, which we have denoted as $t_{N_{s}} / 2$. Thus, at snapshot $s=1$, we have $\widehat{\Omega}_{1}=t_{N_{1}} / 2$, and this implies that a range of values of $\theta$ are selected as admissible, as

${ }^{3}$ The support is the interval $\left(-\Omega_{s}, \Omega_{s}\right)$; we loosely refer to $\Omega_{s}$ as the support, as well. 

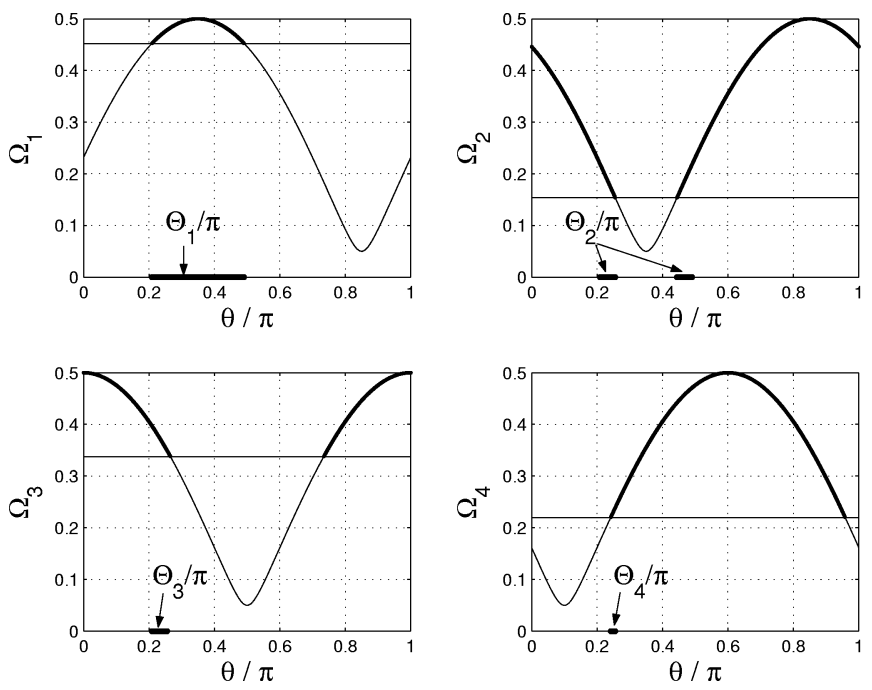

Fig. 3. Rationale of support-based DOA estimation. The four panels show the behavior of $\Omega_{s}(\theta)$ versus $\theta$, at snapshots $s=1,2,3,4$. Consider $s=1$ (top-left panel): the horizontal line represents the support estimation, say $\widehat{\Omega}_{1}$, computed at the first snapshot. The portion of the curve marked in bold highlights the region such that $\Omega_{1} \geq \widehat{\Omega}_{1}$ that defines the range of the admissible values of $\theta$. This range, denoted by $\Theta_{1}$ is marked on the horizontal axis in bold. Consider, hence, the successive snapshot $s=2$ (top-right panel): the curve $\Omega_{2}(\theta)$ is shifted with respect to $\Omega_{1}(\theta)$ because the rover orientation $\phi$ has changed. As before, the horizontal line is drawn in correspondence of $\widehat{\Omega}_{2}$ and defines the region such that $\Omega_{2} \geq \widehat{\Omega}_{2}$, i.e., the range of the admissible values of $\theta$ according to the observations collected at $s=2$. However, not all these values were also admissible at the previous snapshot. $\Theta_{2}$, the jointly admissible values for $s=1,2$, marked in bold on the horizontal axis of the top-right panel, results from the intersection between the $\theta$ admissible at $s=2$, and those previously admissible. The bottom panels refer to $s=3$ (left) and $s=4$ (right) and similar comments apply. In this example, the true DOA is $\theta=\pi / 4$ while $r / v=1$ (arbitrary units) and $h / v=0.1$.

detailed in the caption of Fig. 3. Let us denote such a range with $\Theta_{1}^{\star}$. At the next snapshot, we have $\widehat{\Omega}_{2}=t_{N_{2}} / 2$, and a new range $\Theta_{2}^{\star}$ of admissible DOAs is found. However, only $\Theta_{2}=\Theta_{2}^{\star} \bigcap \Theta_{1}^{\star}$ is admissible after the second snapshot. Continuing, we eventually have $\Theta_{M}=\bigcap_{s=1}^{M} \Theta_{s}^{\star}$ (see again Fig. 3). The boundary of the interval $\Theta_{s}^{\star}$, relevant to the computation of $\Theta_{M}$, can be simply obtained enforcing the equality $\Omega_{s}(\theta)=\widehat{\Omega}_{s}$, with $\Omega_{s}(\theta)$ given by (2). Solving with respect to $\sin ^{2}\left(\theta-\phi_{s}\right)$ provides us with the extremal points $\left[\phi_{s} \pm \arcsin \sqrt{1-\left(2 v \widehat{\Omega}_{s} / r\right)^{2} / 1-(h / r)^{2}}\right] \bmod \pi$.

We have thus interval estimators of the DOA; if a point estimate, say $\widehat{\theta}_{\text {supp }}$, is desired, then we can select an average value. ${ }^{4}$ Note that $t_{N_{s}} / 2$ always yields a lower bound of the true support, meaning it is a biased (from below) estimator of $\Omega_{s}$. Thus, using $t_{N_{s}} / 2$ allows us to exclude DOAs incompatible with the measured data. Note also that in defining the estimator, we have not considered the functional form of the pdf, only its domain of support. As to the physical parameters relevant to our problem, it should be clear that the larger $N_{s}$, the better $t_{N_{s}} / 2$ estimates $\Omega_{s}$. Accordingly, the larger the average number of sensors inside the rover's lobe, the better the system works: We want $\lambda r$ and $h$ as large as possible, and we encapsulate this as the expected number of sensors in a rover's field of view, $N_{\text {lobe }}=\lambda \pi r h / 4$. It can be also shown, and is perhaps intuitive, that better performances are expected with greater ellipse eccentricities, i.e., with smaller $a=h / r$. It is also obvious that the estimate error reduces by increasing the number $M$ of total (independent) snapshots taken.

${ }^{4}$ Actually, $\Theta_{s}$ is not necessarily a single interval; see, for instance, $\Theta_{s=2}$ in Fig. 3; should $\Theta_{M}$ be multiply-connected, we select one interval at random for our estimation task.

\section{MAXIMUM-LIKELIHOOD DOA ESTIMATION}

A different estimation procedure is now described that does use the density of the observables. Recall that $\tau_{s}$ is made of the $\tau_{i}$ 's arranged in increasing order, so that we have $\tau_{s} \sim f_{\tau_{s}}\left(\tau_{s} ; \Omega_{s}\right)=$ $N_{s} ! \prod_{i=1}^{N_{s}} f_{\tau_{i}}\left(\tau_{i} ; \Omega_{s}\right)$, in the range $-\Omega_{s} \leq \tau_{1} \leq \tau_{2} \leq \ldots \leq \tau_{N_{s}} \leq$ $\Omega_{s}$, and zero otherwise. Here, $f_{\tau_{i}}\left(\tau_{i} ; \Omega_{s}\right)$ is the pdf given in (1), and the symbol $\sim$ is used to mean that the random vector is distributed according to that joint pdf. Recall also that the $\tau_{i}$ 's pertinent to a single snapshot are independent and identically distributed (i.i.d.), and we have used this property in the above. Times collected in successive snapshots are independent, but not identically distributed, as (1) depends on $\phi_{s}$.

As noted earlier, we cannot observe the absolute times $\tau_{s}$, but only the time differences $t_{s}$. Now consider the $N_{s}$ random variables $\left(\tau_{1}, t_{2}, t_{3}, \ldots, t_{N_{s}}\right)=\left[\tau_{1}, \boldsymbol{t}_{s}\right]$, with joint density [4]

$$
\begin{aligned}
F\left(\tau_{1}\right) & =f_{\left[\tau_{1}, \boldsymbol{t}_{s}\right]}\left(\tau_{1}, t_{2}, t_{3}, \ldots, t_{N_{s}} ; \Omega_{s}\right) \\
& =f_{\tau_{s}}\left(\tau_{1}, t_{2}+\tau_{1}, t_{3}+\tau_{1}, \ldots, t_{N_{s}}+\tau_{1} ; \Omega_{s}\right) \\
& =N_{s} !\left(\frac{2}{\pi \Omega_{s}}\right)^{N_{s}} \prod_{i=1}^{N_{s}} \sqrt{1-\left(\frac{t_{i}+\tau_{1}}{\Omega_{s}}\right)^{2}}
\end{aligned}
$$

provided that all $t_{i}$ 's are nonnegative and that $-\Omega_{s} \leq \tau_{1} \leq \Omega_{s}-t_{N_{s}}$, and zero otherwise (we have also set $t_{1}=0$ ). Integrating out $\tau_{1}$ yields the density of $\boldsymbol{t}_{s}$

$$
f_{\boldsymbol{t}_{s}}\left(\boldsymbol{t}_{s} ; \Omega_{s}\right)=\int_{-\Omega_{s}}^{\Omega_{s}-t_{N_{s}}} F\left(\tau_{1}\right) d \tau_{1}
$$

that we seek. The above integral, which implicitly depends upon the DOA $\theta$, cannot be evaluated analytically, and in the Appendix, we provide an approximate solution. Once $f_{\boldsymbol{t}_{s}}\left(\boldsymbol{t}_{s} ; \Omega_{s}\right)$ is computed, the likelihood pertinent to $M$ independent snapshots is the product of the individual likelihoods. Accordingly, we have

$$
\widehat{\theta}_{\mathrm{ML}}=\arg \max _{\theta} \prod_{s=1}^{M} f_{\boldsymbol{t}_{s}}\left(\boldsymbol{t}_{s} ; \Omega_{s}\right)
$$

as the ML DOA estimate, and it is probably worth noting that (4) describes the observation pdf conditioned on $N_{s}$, the number of returns (beeps). An overall likelihood function would be a product of this with a probability mass function on $N_{s}$-generally, we would take this as Poisson. However, since the number of returns is a function of the area in the rover's field of view, and not of its orientation, we do not need to consider that term in our DOA estimation.

\section{RESULTS}

Fig. 4 shows the mean-square errors (MSEs) of the two estimators $\widehat{\theta}_{\text {supp }}$ and $\widehat{\theta}_{\mathrm{ML}}$, plotted against the total snapshot number $M$. The MSEs are computed according to a standard Monte Carlo counting approach, i.e., we have run many independent computer experiments; for each such run, the proposed estimators are implemented and the pertinent squared errors evaluated. These data are then averaged over the many runs, ending up with the MSEs shown in the figure. ${ }^{5}$

For comparison, the asymptotic MSE of the simple DOA estimator proposed in [1] is also given (see the curve labeled with "prev," meaning previous). As to the latter, the curve is actually the Fisher information and represents the asymptotically achievable MSE. ${ }^{6}$

${ }^{5}$ For generality, in the computer experiments performed, different Monte Carlo runs use different sequences $\left\{\phi_{s}, s=1,2, \ldots\right\}$ of rover orientations.

${ }^{6}$ See [1] for Cramer-Rao lower bound (CRLB) details. It would be preferable to develop and compare results with the CRLB in the new schemes, as well. Unfortunately, the technical conditions usually required for the CRLB's validity [5] are violated: The parameter to be estimated determines the support of the pdf. 

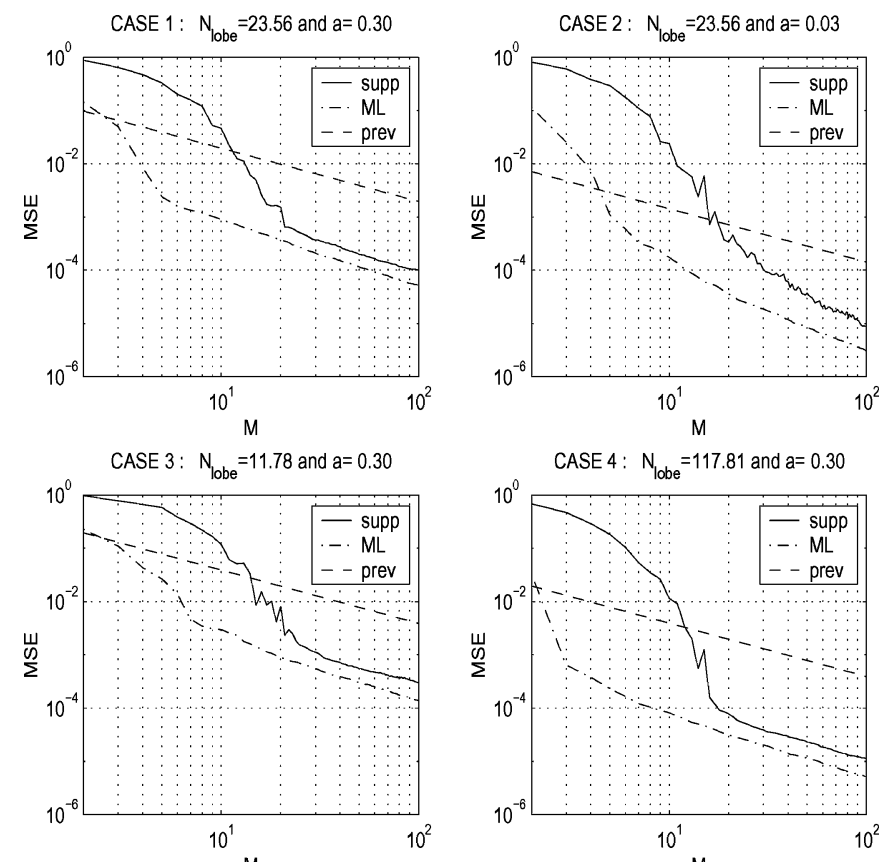

Fig. 4. Comparison between the MSEs of $\widehat{\theta}_{\text {supp }}$ and $\widehat{\theta}_{\mathrm{ML}}{ }^{\mathrm{M}}$, and that of the estimator proposed in [1] ("prev"), for which the theoretical asymptotic MSE is shown. The four cases considered reproduce the scenarios addressed in [1]. Case 1 assumes $\lambda=100, r=1$, and $h=0.3$ (in arbitrary measure units); in case 2 , we have $\lambda=10, r=10$, and $h=0.3$; case 3 refers to $\lambda=50, r=1$, and $h=0.3$; finally, in case 4 , we set $\lambda=500, r=1$, and $h=0.3$. In the figures, $N_{\text {lobe }}$ is the expected number of samples in the rover's field of view, and $a$ is the ratio of the minor to major axis lengths for this assumed-elliptical pattern.

When compared with the previously proposed estimator, the performance improvements obtained with $\widehat{\theta}_{\text {supp }}$ and $\widehat{\theta}_{\mathrm{ML}}$ are impressive: More than an order of magnitude may be gained after approximately 100 snapshots. Comparing $\widehat{\theta}_{\text {supp }}$ to $\widehat{\theta}_{\mathrm{ML}}$, it is not unexpected that the ML method always outperforms. However, usually there is little difference, at least with $M \in(30,100)$.

As to the computational burden of the three estimators, the method presented in [1] basically counts the beeps in a given strip, inserts this number in a proper likelihood function, and numerically finds the maximum over $\theta$, perhaps by computing the likelihood over a sufficiently dense grid of values of $\theta$. The ML approach introduced in this correspondence needs a numerical implementation of the maximization of a certain likelihood function, while the support based is much simpler because it essentially relies upon computing successive intersections of allowable ranges, as discussed in Section II. To get an approximate idea of the numbers involved, we have used the floating-point operations (FLOPS) counting routine built in Matlab to estimate the computational load, using as testbeds the four applicative scenarios of Fig. 4. For any snapshot, the method presented in [1] requires $\approx 10^{4}$ FLOPS, and this number stays essentially constant for the four cases of Fig. 4. The ML estimator may be computed in $\approx 10^{6}$ to $10^{7}$ FLOPS, with larger burdens associated with larger values of $N_{\text {lobe }}$. Finally, the support-based requires only $\approx 50$ FLOPS, in all the four cases. These numbers, obviously, may change with application and platform, but there is little doubt that the support-based scheme is quite appealing in its low numerical burden.

\section{SUMMARY AND DISCUSSION}

Can DOA be inferred from a field of sensors, despite no knowledge of individual sensor identities, locations, or number, and despite each sensor being completely isotropic in its sensing ability? Let us assume the SENMA paradigm in which a mobile rover polls from the field: DOA information is contained in periodic emanations (notionally: beeps), each triggered by the passage of the wavefront of interest through a sensor. The rover observes these emanations from interrogations of multiple polled areas, and it is key to success that these fields of view be eccentric.

The essence of a previous paper [1] was to count the number of sensors within a central strip of the polling area. In this correspondence, we use fuller information: We measure the distribution of sensors, as observed by their (unlabeled) emanations. Two approaches have been developed - one somewhat involved and based on maximizing the likelihood, and the other simple and using only a support measure of the length of the projection of the ellipse onto the DOA vector. Results show remarkable improvement over the scheme of [1], as much as an order of magnitude reduction in MSE. There is some small difference between their statistical efficiency, but the extreme triviality of the support estimator is highly appealing: The numerical load imposed by support-based estimation at the rover is almost insignificant. Further, since the ML method exploits the analytical expression of the pdf, while the support based does not, it is presumable that $\widehat{\theta}_{\text {supp }}$ might better tolerate model inaccuracies than $\widehat{\theta}_{\mathrm{ML}}$. Neither the ML nor support-based schemes require an assumption (e.g., a Poisson law) on the number of sensors available to a rover poll, since their estimates are posterior to the number of responses; all that is necessary is that those sensors that do report be uniformly distributed throughout the rover's field of view. Since the technique of [1] does require an explicit Poisson law, this may be considered an additional advantage to the new approaches. Put another way, the technique of [1] requires that the Poisson density be known for each rover poll; the techniques here need only a local Poisson assumption and do not require that the Poisson parameter be known beforehand.

In this correspondence, we have made, implicitly or explicitly, certain simplifying assumptions:

- errors in the impinging time measurements of sensors are neglected, and sensors' internal clocks are perfectly synchronized;

- the rover has perfect control of its field of view and in particular it knows exactly the lobe orientation;

- the field of view has a distinct boundary (meaning that a "poll" involves all sensors inside and none outside), while in practice a smoother behavior might be expected.

This last assumption is not particularly important to the ML approach but is critical to the support-based scheme since if we instead assumed that sensors were polled/seen with a certain probability $p, 0<p<1$, then the resulting impinging time density (the equivalent of (1)) would have unbounded support. A suitable approach could perhaps rely on the range of the measured vector (spread between larger and smaller order statistics), and we have ongoing work on this.

Further, issues of sure practical relevance are the presence of inconsistence/absence of sensor measurements due to vanishing acoustic signals (low signal-to-noise ratios) and/or sensors failures, and the effect of multiple impinging wavefronts. We intend also to develop means to allow acoustic (as opposed to the current instantaneous) sensor/rover communication, to consider the efficiency of the various schemes, to allow for (and compute the system-level probability of) false alarms and to localize near-field sources by removing the restriction that impinging waves be planar.

\section{APPENDIX}

An approximate evaluation of the integral in (4) can be derived as follows. With reference to (3), let us set

$$
g\left(\tau_{1}\right)=-\frac{1}{2} \sum_{i=1}^{N_{s}} \log \left[1-\left(\frac{t_{i}+\tau_{1}}{\Omega_{s}}\right)^{2}\right] .
$$


Considering the first terms of the Taylor's series about the point $\tau_{0}$, we get $g\left(\tau_{1}\right) \approx g\left(\tau_{0}\right)+g^{\prime \prime}\left(\tau_{0}\right)\left(\left(\tau_{1}-\tau_{0}\right)^{2} / 2\right)$, where $\tau_{0} \in\left(-\Omega_{s}, \Omega_{s}-\right.$ $\left.t_{N_{s}}\right)$ is such that $g^{\prime}\left(\tau_{0}\right)=0$. This approximation gives

$$
F\left(\tau_{1}\right) \approx N_{s} !\left(\frac{2}{\pi \Omega_{s}}\right)^{N_{s}} e^{-g\left(\tau_{0}\right)} e^{-g^{\prime \prime}\left(\tau_{0}\right) \frac{\left(\tau_{1}-\tau_{0}\right)^{2}}{2}}
$$

from which integration in (4) is easily performed, yielding

$$
\begin{aligned}
f_{\boldsymbol{t}_{s}}\left(\boldsymbol{t}_{s} ; \Omega_{s}\right)= & N_{s} ! \\
\times\{ & \left(\frac{2}{\pi \Omega_{s}}\right)^{N_{s}} e^{-g\left(\tau_{0}\right)} \sqrt{\frac{2 \pi}{g^{\prime \prime}\left(\tau_{0}\right)}} \\
& {\left[\sqrt{g^{\prime \prime}\left(\tau_{0}\right)}\left(-\Omega_{s}-\tau_{0}\right)\right] } \\
& \left.-Q\left[\sqrt{g^{\prime \prime}\left(\tau_{0}\right)}\left(\Omega_{s}-t_{N_{s}}-\tau_{0}\right)\right]\right\} .
\end{aligned}
$$

Here, $Q(\cdot)$ is the standard Gaussian exceedance probability function, and

$$
\begin{aligned}
e^{-g\left(\tau_{0}\right)} & =\prod_{i=1}^{N} \sqrt{1-\left(\frac{t_{i}+\tau_{0}}{\Omega_{s}}\right)^{2}} \\
g^{\prime \prime}\left(\tau_{0}\right) & =\sum_{i=1}^{N_{s}} \frac{\Omega_{s}^{2}+\left(t_{i}+\tau_{0}\right)^{2}}{\left[\Omega_{s}^{2}-\left(t_{i}+\tau_{0}\right)^{2}\right]^{2}} .
\end{aligned}
$$

As said, $\tau_{0}$ is implicitly defined as the solution of $g^{\prime}(x)=0$, viz.

$$
\sum_{i=1}^{N_{s}} \frac{t_{i}+x}{\Omega_{s}^{2}-\left(t_{i}+x\right)^{2}}=0 .
$$

Rather than trying to solve exactly this equation, we content ourselves with a numerical approximate solution, exploiting the fact that $g(\cdot)$ is a convex $\cup$ function over the open interval $\left(-\Omega_{s}, \Omega_{s}-t_{N_{s}}\right)$. In fact, from the well-known Newton-Raphson approach (see, e.g., [6]), we start with a tentative solution $\xi^{(0)}=-t_{N_{s}} / 2$ (that is, the middle point of the interval) and expand $g^{\prime}(x)$ in a small neighborhood thereof: $g^{\prime}(x) \approx$ $g^{\prime}\left(\xi^{(0)}\right)+g^{\prime \prime}\left(\xi^{(0)}\right)\left(x-\xi^{(0)}\right)$. Setting this to zero defines a new tentative solution $\xi^{(1)}$ to be inserted in such equation in place of $\xi^{(0)}$, and so on. We find that a few iterations are usually sufficient, and in fact often just one single iteration may suffice. In this case

$$
\tau_{0} \approx \xi^{(1)}=-\frac{t_{N_{s}}}{2}-\frac{g^{\prime}\left(-\frac{t_{N_{S}}}{2}\right)}{g^{\prime \prime}\left(-\frac{t_{N_{S}}}{2}\right)}
$$

Substitution of such a $\tau_{0}$ (or the value resulting from successive iterations) in (6) provides us with the approximation sought.

\section{REFERENCES}

[1] S. Marano, V. Matta, P. Willett, and L. Tong, "DOA estimation via a network of dumb sensors under the SENMA paradigm," IEEE Signal Process. Lett., vol. 12, no. 10, pp. 709-712, Oct. 2005.

[2] L. Tong, Q. Zhao, and S. Adireddy, "Sensor networks with mobile agents," in Proc. IEEE Military Communications Conf. (MILCOM) 2003, Boston, MA, Oct. 2003, pp. 688-693.

[3] D. L. Snyder and M. I. Miller, Random Point Processes in Time and Space, 2nd ed. New York: Springer-Verlag, 1991.

[4] H. David, Order Statistics. New York: Wiley, 1981.

[5] H. L. Van Trees, Detection, Estimation and Modulation Theory. New York: Wiley, 2001, pt. I, p. 66.

[6] W. T. Vetterling and B. P. Flannery, Numerical Recipes in C++: The Art of Scientific Computing, 2nd ed, W. H. Press and S. A. Teukolsky, Eds. Cambridge, U.K.: Cambridge Univ. Press, 2002, pp. 366-372. 